\title{
Enhanced hyperplasia in muscles of transgenic zebrafish expressing Follistatin1
}

\author{
LI Xi, NIE Fen, YIN Zhan \& HE JiangYan* \\ Institute of Hydrobiology, Chinese Academy of Sciences, Wuhan 430072, China
}

Received May 25, 2010; accepted October 22, 2010

\begin{abstract}
Myostatin is a member of the transforming growth factor- $\beta$ (TGF- $\beta$ ) super-family and functions as a negative regulator of muscle growth. Binding of the specific receptor, Activin receptor IIB (Act RIIB), with myostatin or other related TGF- $\beta$ members, could be inhibited by the activin-binding protein follistatin (Fst) in mammals. Overexpressing Fst in mouse skeletal muscle leads to muscle hypertrophy and hyperplasia. To determine if $F s t$ has similar roles in fish, we generated transgenic zebrafish expressing high levels of zebrafish Fst 1 using the promoter of the zebrafish skeletal muscle-specific gene, myosin, light polypeptide 2, skeletal muscle (Mylz2). Independent transgenic zebrafish lines exhibited elevated expression levels of myogenic regulatory genes $M y o D$ and $\operatorname{Pax} 7$ in muscle cells. Adult Fst1 overexpressing transgenic zebrafish exhibited a slight body weight increase. The high level of $F$ st 1 expression dramatically increased myofiber numbers in skeletal muscle, without significantly changing the fiber size. Our findings suggest that $F s t 1$ overexpression can promote zebrafish muscle growth by enhancing myofiber hyperplasia.
\end{abstract}

Follistatin1, myostatin, muscle growth, transgenic, zebrafish

Citation: Li X, Nie F, Yin Z, et al. Enhanced hyperplasia in muscles of transgenic zebrafish expressing Follistatin1. Sci China Life Sci, 2011, 54: 159-165, doi: $10.1007 / \mathrm{s} 11427-010-4121-2$

Myostatin, a member of the transforming growth factor super-family, has been shown to negatively regulate satellite cell growth and post-natal myogenesis through Pax7 signals [1]. Like other members of the TGF- $\beta$ superfamily, myostatin is synthesized as a precursor protein consisting of a signal peptide, an amino-terminal propeptide domain (or prodomain) and a carboxy-terminal mature (active) domain. The precursor undergoes two proteolytic processing events by its proprotein convertases to generate the biologically active molecule. The precursor protein forms a homodimer before proteolytic processing. The cleaved propeptide molecule remains non-covalently bound to the mature domain dimer, forming a latent complex, which inhibits its biological activity by inhibiting myostatin binding to its receptor. It has been reported that myostatin exhibits a pivotal role as a

*Corresponding author (email: jyhe@ihb.ac.cn) negative regulator of skeletal growth in zebrafish, as do its orthologs in higher vertebrates. In addition, transgenic zebrafish expressing the myostatin prodomain could increase the number of fibers in skeletal muscle [2-4].

Follistatin (Fst) is a secreted glycoprotein that was first identified as a potent inhibitor of some members of the TGF- $\beta$ super-family, because of its strong binding affinity for the receptor protein, activin [5,6]. It can also directly bind some TGF- $\beta$ ligand proteins at lower affinities compared to activin in mammals [6-8]. Fst can block the activity of growth differentiation factor (GDF-11) and myostatin via competitive binding. Fst knockout mice have reduced muscle mass at birth [9], whereas mice overexpressing Fst in skeletal muscle showed a gross increase in muscle fiber hyperplasia and hypertrophy, compared to wild-type animals [10,11].

The cyprinid zebrafish (Danio rerio) was recently shown 
to have two Fst genes, Fst1 and Fst2. Fst1 is universal among teleosts, and is closely related to the single gene of higher vertebrates. During the embryonic stage, zebrafish Fst1 is initially expressed in the anterior somite before becoming restricted to the external cell layer. Furthermore, Fst 1 is strongly expressed in the ventral region of rostral somites, which produces presumptive myogenic precursors of muscles, suggesting a potential role of Fstl in myogenesis in cyprinids [12].

Fst's inhibitory function on GDFs and the enhanced muscle growth phenotypes caused by the overexpression of Fst in mice, prompted us to produce Fst 1 overexpressing zebrafish, with the Fst 1 driven by the mylz2 promoter, a strong zebrafish skeletal muscle-specific promoter [13]. The Fst 1 transgenic zebrafish had only a slightly increased body weight; however, Fstl overexpression indeed enhanced $M y o D$ and $\operatorname{Pax} 7$ expression in adult transgenic fish skeletal muscle. Furthermore, skeletal muscle growth of the transgenic zebrafish overexpressing Fstl was significantly enhanced, with a significant increase in numbers of myofibers. Thus, a negative regulatory role for Fst in myostatin-related hyperplasia of muscle in cyprinids is suggested, similar to its orthologs in amniotes.

\section{Materials and methods}

\subsection{Transgenic construction}

The zebrafish mylz2:EGFP transgenic construct was previously described and kindly provided by Dr. Gong ZhiYuan from National University of Singapore [13]. The Tol2 enhancer trap vector described previously was kindly provided by Dr. Korzh V. of the Institute of Molecular and Cellular Biology in Singapore [14]. A fragment containing the multiple cloning site (MCS) and SV40 polyadenylation sequence from pCMV-HA vector (Clontech, PT3285, bp829-1049) was amplified using forward primer (5'-ggatccaccatgtacccatacgatgttccagattacgct- $\left.3^{\prime}\right)$ and reverse primer (5'-ggatcctttattgcagcttataatggtt- $\left.3^{\prime}\right)$, which both contain a $B a m \mathrm{H}$ I recognition site (underlined). The amplified fragment was then cloned into Bam H I-digested mylz2:EGFP to make our muscle expression intermediate vector. After a series of $X b a$ I digestion, filling-in with the Klenow fragment and self-ligation, we gradually destroyed the $X b a$ I site within our intermediate construct. The fragment containing the mylz2 promoter region, the MCS, and SV40 polyadenylation sequence was then amplified with the forward primer (5'-aattcgccacagaggaatg-3') and reverse primer (5'tttattgcagcttataatggtt- $3^{\prime}$ ), both containing an Xba I recognition sites. The amplified fragment was then cloned into Xba I-digested Tol 2 enhancer trap vector to make our muscle specific promoter, and keratins basal promoter driven EGFP expression vector (MHK vector). This MHK vector contains an MCS originally from pCMV-HA vector. Finally, a full-length cDNA of zebrafish $F$ stl was cloned via RT-PCR from a zebrafish embryonic total RNA sample. Two oligonucleotide primers, forward primer (5'-atgaattcggatgctaaggatgctaaagcg-3') containing a EcoR I recognition site (underlined), and reverse primer (5'-atctcgagctacttgtcgtc atcgtcttgtagtc ggcgcacacagcttcctccg- $3^{\prime}$ ) containing a Xho I recognition site (underlined) and a tag peptide coding region (italic), were used to amplify a $0.9 \mathrm{~kb}$ of $F$ stl coding region based on the zebrafish Fst1 mRNA deposited in GenBank (Accession No. NM_131037). The amplified Fst1 products were cloned into the MHK vector, where Fst1 expression was driven by the zebrafish mylz2 promoter. The SV40 polyadenylation sequence for transcription termination of Fst1 expression was followed by an epithelial specific EGFP expression unit controlled by zebrafish keratins promoter, which could be used as a linked reporter gene product for positive gene integration screening (Figure 1A). The Tol2 arms flanking the Mylz2 promoter, zebrafish Fst 1 coding region, SV40 polyadenylation sequence, keratins promoter, EGFP coding region and SV40 polyadenylation sequence were used to enhance genome integration [14].

\subsection{Production of transgenic fish}

The medaka transposase cDNA clone [14] was kindly provided by Dr. Korzh V. Ten nanograms of MHK-Fst1 plasmid DNA with $25 \mathrm{ng}$ of in vitro synthesized transposase mRNA was co-injected into zebrafish embryos at the one- or two-cell stage, as previously described by Parinov et al. [14]. Expression of GFP in the injected embryos was observed and photographed under a Leica fluorescence microscope.

Injected embryos with epithelial EGFP expression were allowed to grow and cross with wild-type fish. For each F1 cross, at least 100 embryos were examined under a fluorescence microscope. The presence of the transgene fragment was tested by PCR amplification using fish genomic DNA as the template and forward primer (within the mylz promoter region) 5'-TCAGTGGCTACAGCTCATTCA-3' and reverse primer (within the zfFst1 coding region) 5'TTTGTCTGGTCCACCACGCAA-3'. The expected size of the amplified fragment was $981 \mathrm{bp}$. The identified transgenic founders were then crossed with wild-type fish for the analysis.

\subsection{Whole-mount in situ hybridization}

Whole-mount in situ hybridization using a digoxigenin (DIG)-labeled riboprobe was carried out as previously described [15]. The zebrafish Fstl cDNA clone was amplified as above and cloned into pBluescript vector [13]. The plasmid was linearized with Bam $\mathrm{H}$, followed by an in vitro transcription reaction with T7 RNA polymerase to generate the antisense RNA probe. The embryos/larvae up to five days post fertilization (dpf) were fixed in $4 \%$ paraformaldehyde, and hybridized with the DIG-labeled riboprobe in 


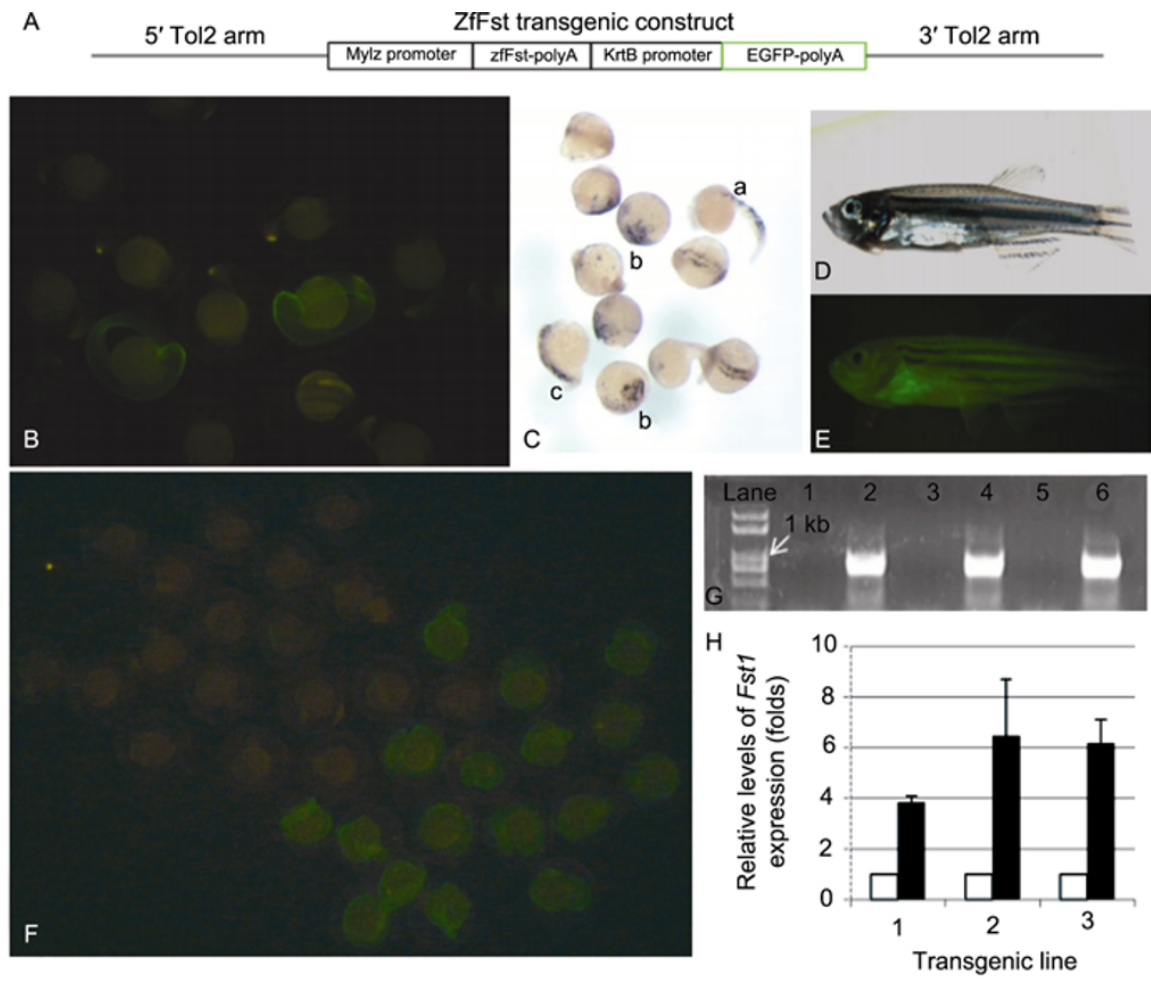

Figure 1 Generation of transgenic zebrafish. A, Transgenic construct containing 5' and 3' ends of Tol2 recombination arms at each end of the functional region. The functional region contains a $1.9 \mathrm{~kb}$ zebrafish myosin light polypeptide 2 (mylz2) promoter, the $0.9 \mathrm{~kb}$ zebrafish follistatin 1 (Fst1) coding region and SV 40 poly(A) signal, a $0.5 \mathrm{~kb}$ basal promoter of zebrafish keratin 8 (krt8), the $0.8 \mathrm{~kb}$ EGFP coding region and a SV 40 poly(A) signal. B, Fluorescence image of MHK-Fst1 injected embryos observed at $24 \mathrm{hpf}$. The embryos with EGFP expression in the skin indicated the successful delivery of the transgenic construct via microinjection. C, Fst1 expression observed in embryos injected with MHK-Fst1 at 48 hpf stages via whole mount in situ hybridization. Most of the Fstl overexpression caused defects during embryonic development (as indicated with "b", or "c"), while only a small proportion of injected embryos showed normal development (as indicated with "a"). D and E, Images of a transgenic founder under normal microscopy (D) and fluorescence microscopy (E). F, Fluorescence image of a batch of embryos from the cross between a wild-type and a stable transgenic founder. Embryonic expression of EGFP in the skin suggested a stable germline transmission. G, Insertion test using genomic DNA PCR tests. Amplified PCR fragments of the correct size and sequence are shown in lanes 2, 4, and 6 representing stable transgenic lines 1, 2, and 3. Their non-insertion containing control sibling DNAs are in lanes 1 , 3, and 5. H, Comparison of Fst 1 overexpression in skeletal muscle between transgenic zebrafish and controls at 60 dpf via real-time PCR. The data represents three replicates in each transgenic line. a, transgenic larva with a normal development; b, dorsalizing patterning of some MHK-Fst linjected embryos; c, cessation of embryonic development in some MHK-Fst1 injected embryos.

hybridization buffer [50\% formamide, $5 \times \mathrm{SSC}(1 \times=15 \mathrm{mmol}$ $\mathrm{L}^{-1} \mathrm{NaCl}, 15 \mathrm{mmol} \mathrm{L}^{-1}$ sodium citrate, $\left.\mathrm{pH} 7.6\right), 50 \mathrm{mg}$ heparin, $50 \mathrm{mg} \mathrm{mL}^{-1}$ tRNA and $0.1 \%$ Tween] at $70^{\circ} \mathrm{C}$. They were then incubated with anti-DIG antibody conjugated with alkaline phosphatase and stained with nitroblue phosphate (NBT) and 5-bromo, 4-chloro, 3-indolil phosphate (BCIP) to produce purple and insoluble precipitates.

\subsection{RNA isolation and real-time PCR}

The levels of Fst1, MyoD and Pax7 in non-transgenic control or Fst1 transgenic fish were quantitatively analyzed using real-time PCR at $60 \mathrm{dpf}$. About $100 \mathrm{mg}$ of muscle tissue was used for total RNA extraction with Trizol reagent, following the procedure recommended by the manufacturer (Invitrogen). The total RNA was digested with RNase-free DNAase to remove endogenous DNA contamination. Firststrand cDNA was synthesized by reverse transcriptase using a SuperScript ${ }^{\circledR}$ III platinum One-step qRT-PCR Kit (Invitrogen). Zebrafish Fst1, MyoD, and $\operatorname{Pax} 7$ were detected by amplification using gene-specific primers (Table 1). Zebrafish $\beta$-actin gene expression was also analyzed (producing a 102 bp amplified product) as an internal control using specific primers (Table 1). All amplicons were sequenced to confirm the desired products.

Real-time PCR was carried out on a Sequence Detector (PRISM 7700; ABI, Foster City, CA, USA). Each reaction contained $2 \mu \mathrm{L}$ cDNA and primers at a final concentration of $2 \mathrm{ng} \mu \mathrm{L}^{-1}$. The samples were first heated to $50^{\circ} \mathrm{C}$ for $2 \mathrm{~min}$, followed by $95^{\circ} \mathrm{C}$ for $10 \mathrm{~min}$. The PCR reaction was carried out for 40 cycles of $95^{\circ} \mathrm{C}$ for $15 \mathrm{~s}$ and $60^{\circ} \mathrm{C}$ for $1 \mathrm{~min}$. SDS $\mathrm{v} 1.7 \mathrm{a}$ software was used to define the cycle in which each sample attained the threshold value. All values were computed using a standard curve and normalized to zebrafish $\beta$-actin, which served the internal positive control. 
Table 1 Primers for real-time RT PCR

\begin{tabular}{|c|c|c|c|}
\hline Gene & Primer & Primer sequence & Size of amplicon \\
\hline \multirow[t]{2}{*}{ Zebrafish Fst1 } & Forward & 5'-GACGTGTGATAATGTTGACTGTGG-3' & $214 \mathrm{bp}$ \\
\hline & Reverse & 5'-GCATTTGCCTTGATACTGGACCTC-3' & \\
\hline \multirow[t]{2}{*}{ Zebrafish $M y o D$} & Forward & 5'-CGCCATTAGTTATATCGAGTC-3' & $200 \mathrm{bp}$ \\
\hline & Reverse & 5'-CGTCATTGAAGTAAGAGCTG-3' & \\
\hline \multirow[t]{2}{*}{ Zebrafish $\operatorname{Pax} 7$} & Forward & 5'-ATAAGTTGCTGAAGGACGGAGTGT-3' & $127 \mathrm{bp}$ \\
\hline & Reverse & 5'-TCACACTCGTCATCGTCATCTTTC-3' & \\
\hline Zebrafish $\beta$-actin & Reverse & 5'-CAACGGAAACGCTCATTGC-3' & \\
\hline
\end{tabular}

\subsection{Growth evaluations and muscle histological analy-} sis

To determine their body weights, all fish were weighted at 30 and $60 \mathrm{dpf}$. For muscle histological analysis, muscle tissues from ten individual fish from each group at $60 \mathrm{dpf}$ were fixed in Bouin's solution for $10 \mathrm{~h}$ followed by routine paraffin sectioning and Hematoxilin/Eosin staining. Vertical sections at the base of the first pin of the pelvic fin were selected for quantification of the muscle fiber number.

\section{Results}

\subsection{Generation of transgenic zebrafish}

The MHK-Fst1 plasmid constructs were co-injected with the synthesized medaka transposase mRNA into fish embryos. Seventy-five percent of the injected embryos showed abnormal developmental features and died prior to hatching, possibly as a result of mis-expression of Fstl during embryonic stages (a representative features of MHK-Fst1 injected F0 embryos are shown in Figure 1C, labeled as a, b, c). However, 45 fish survived to adulthood from among the 180 injected embryos (Figures 1D and E). The transgenic founders (F0) were initially screened based on the epithelial EGFP-expression observed under fluorescence microscopy (Figures 1B and E). As a result, we obtained 28 F0 fish showing epithelial EGFP expression, and three of them showed germline transmission. Skeletal muscle Fst1-expression was confirmed using a genomic DNA PCR test to ensure the correct insertion within the zebrafish genome. (Figure 1G). These germline transmitters were then used to produce $\mathrm{F} 1$ generations. The transmission rate of the transgene into the F1 generation varied from 3\%-7\% (Figure 1F). Unlike initial embryos injected with the mylz:fst1 construct, the survival rates of all F1 transgenic strains were not significantly difference from those of non-transgenic fish (Figures $1 \mathrm{E}$ and $\mathrm{F}$ ). Using real-time PCR, we confirmed that the average relative expression level of Fstl in $60 \mathrm{dpf}$ transgenic fish skeletal muscle tissue was much higher than that in control zebrafish (Figure 1H): about six-fold higher in lines 2 and 3 than in their non-transgenic control sibling fish.

\subsection{Fish body weight}

To determine body weight, we weighed fish at 30 and 60 dpf and compared transgenic fish with their control siblings. At $30 \mathrm{dpf}$, the body weights of the transgenic fish and the controls were not significantly different. However, at $60 \mathrm{dpf}$, the average body weight of our Fst1 hemizygous transgenic zebrafish had increased slightly, but significantly (by $8.14 \%$ ), compared with their non-transgenic sibling controls (Table 2). The observed phenotype was more obvious compared that observed in hemizygous myostatin prodomain transgenic zebrafish, who showed no significant enhancement in body mass [2]. This suggests that Fstl overexpression has a stronger influence on body mass increase than myostatin prodomain overexpression.

\subsection{Myogenic gene expression levels in Fst1 overexpre- ssing transgenic zebrafish}

To determine if Fstl overexpression enhances the expression of a myogenic regulatory gene, we analyzed the expression of $M y o D$ in transgenic fish embryos by whole mount in situ hybridization. There was no significant change in $M y o D$ expression in transgenic zebrafish at embryonic stages (data not shown). However, at later stages (60 dpf), we analyzed the expression of $M y o D$ and $\operatorname{Pax} 7$ in transgenic zebrafish by quantitative real time RT-PCR. The expression level of $M y o D$ was significantly upregulated in Fst 1 adult transgenic zebrafish skeletal muscle tissue compared with its non-transgenic siblings (Figure 2). As $M y o D$, Pax7 appears to have critical roles as well during satellite cell activation, proliferation, and differentiation. The expression of Pax7 in fish muscle was enhanced in the Fstl overexpressing zebrafish muscle at $60 \mathrm{dpf}$ stage (Figure 2). This result indicated that Fstl overexpression in skeletal muscle could enhance myogenesis in zebrafish.

\subsection{Histological analysis of muscle growth}

To determine whether or not muscle growth was affected in the Fst 1 transgenic fish, samples of representative transgenic fish and their non-transgenic siblings were sectioned to determine fiber size and number at $60 \mathrm{dpf}$ (Figures 3A and $\mathrm{B})$. The number of myofibers from a defined region of the epaxial muscle at the base of the first pin of the pelvic 
Table 2 Body mass comparison between transgenic fish and non-transgenic siblings

\begin{tabular}{|c|c|c|c|c|c|}
\hline \multirow{2}{*}{ Age } & \multicolumn{2}{|c|}{ Control fish } & \multicolumn{2}{|c|}{ Fst transgenic fish } & \multirow{2}{*}{$P$-value ${ }^{\text {b) }}$} \\
\hline & Number & Mass $(g)^{a)}$ & Number & Mass $(\mathrm{g})^{\mathrm{a})}$ & \\
\hline $30 \mathrm{dpf}$ & 11 & $0.03091 \pm 0.01004$ & 4 & $0.02950 \pm 0.01489$ & $0.05346(>0.05)$ \\
\hline $60 \mathrm{dpf}$ & 14 & $0.06957 \pm 0.02667$ & 13 & $0.07523 \pm 0.01845$ & $0.2652(<0.05)$ \\
\hline
\end{tabular}

a) Mass is shown as means \pm SE; b) $P$ values are the result of a $t$-test. $P<0.05$ indicates a significant difference.

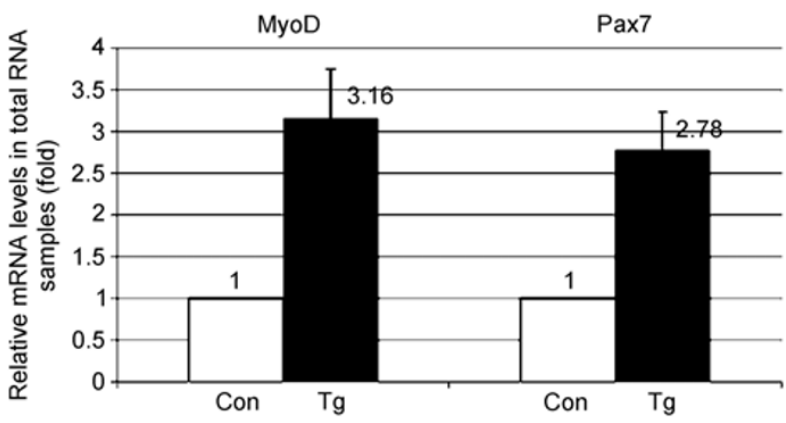

Figure 2 Comparison of myogenic gene $M y o D$ and $\operatorname{Pax} 7$ expression in muscles of transgenic and control fish by real-time PCR. Total RNA was extracted from muscle tissue and real-time PCR was performed as described in Materials and methods. Three individuals from each of transgenic line 2 and 3 were analyzed. The amount of $M y o D$ and Pax7 mRNA analyzed was normalized to $\beta$-actin expression levels. Data are means \pm SD of transgenic and their non-transgenic sibling fish performed in triplicate. The levels of the MyoD and Pax7 expression are statistically significantly different as determined by a $t$-test.

fin was determined by counting them under a microscope at a fixed magnification (Figures 3C, D, E, F, and G). The average number of fibers in Fst1 transgenic fish ((604.14 \pm 47.76), $n=10$ ) was much larger than the corresponding number in non-transgenic control fish $((462.63 \pm 32.12)$, $n=10)$. While a larger number of the myofibers observed in the same region of the muscle tissue of transgenic fish than the ones in control fish, the existence of many small diameter fibers has also been seen obviously in the Fstl transgenic muscle tissue (Figures 3D, E, F, and G). The average individual myofiber area from Fst 1 transgenic zebrafish was not significantly larger than the same area from their control siblings. These results indicated that the enhanced muscle growth induced by Fstl overexpression in zebrafish was caused by hyperplastic growth of muscle, not by hypertrophy.

\section{Discussion}

In this study, an Fst 1 expression construct under the control of the fish skeletal muscle specific promoter, mylz2, was used to investigate the regulatory function of Fst1 in fish muscle development and its potential role in the regulation of fish muscle growth. Microinjection of the mylz2:fst1 construct initially caused a large proportion $(75 \%)$ of malformations during early embryonic development, which might reflect the critical function of the Fst1 in dorsoventral patterning during zebrafish gastrulation [16]. The remaining
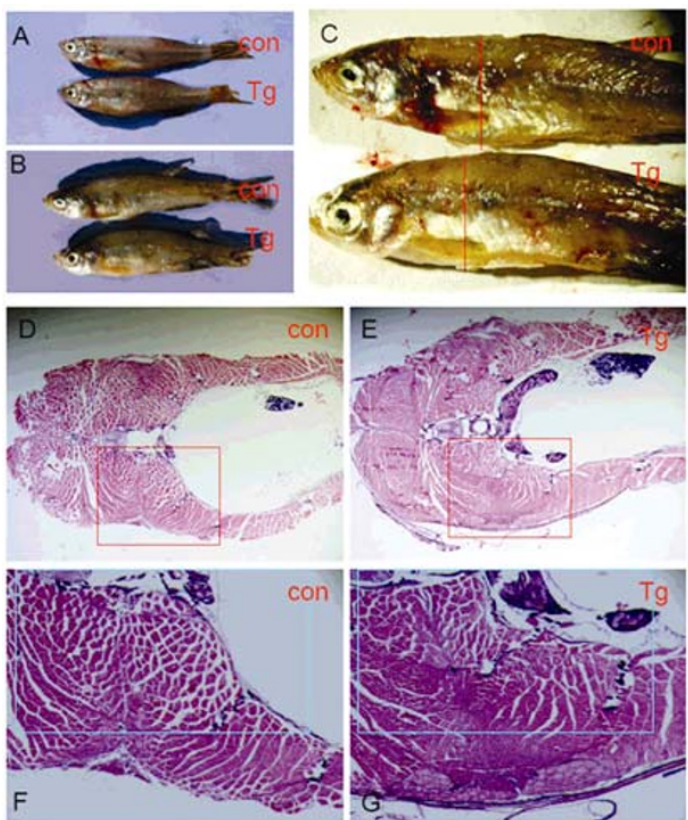

Figure 3 Enhanced muscle growth via hyperplasia in Fst1 overexpression transgenic zebrafish. A and B, Pictures of killed and skinned transgenic ( $\mathrm{Tg}$ ) and their non-transgenic control sibling (con) zebrafish. The Fst1 overexpressing transgenic fish show the typical morphological feature of high and thick muscle tissue in the dorsal muscle region compared with their non-transgenic siblings. C, High magnification image showing the typical morphological feature of the Fst 1 overexpressing transgenic fish. D and E, Low magnification pictures of cross-sections stained with hematoxylin and eosin of the transgenic (E) and control fish (D). F and G, High magnification images of the tissue region in the box indicated in panel of $\mathrm{D}$, E. Histological analysis of Fst 1 over-expressing fish indicated an increasing number of myofibers in transgenic fish compared with their nontransgenic control siblings, based on the count and analysis of the number of myofibers in the box in panels $\mathrm{F}$ and $\mathrm{G}$.

$25 \%$ of the mylz:fst1 injected embryos survived to adulthood, which might be because of the late and muscle-specific expression properties of the mylz2 promoter. Our results revealed that muscle-specific Fstl overexpression significantly enhanced fish muscling (Figure 3). The overexpression of Fst 1 also stimulated MyoD and Pax7 expression in the skeletal muscle of transgenic fish (Figure 2). Histological analysis also suggested that enhanced skeletal muscle growth in the Fstl-overexpressing fish mainly resulted from myofiber hyperplasia (Figure 3 ).

One member of the TGF- $\beta$ superfamily that has a major role in regulating muscle growth is myostatin. In mammals, homozygous deficiency of myostatin causes an impressive increase in muscle mass. Previously, myostatin activity ablation in zebrafish, via RNAi and morpholino injection, also 
led to a giant phenotype and the upregulation of some key myogenic transcriptional factors during embryonic somitogenesis $[3,4]$. However, similarly to a previous transgenic study of myostatin prodomain overexpression [2], we failed to observe any obvious phenotypic differences or upregulation of $M y o D$ and myf5 in our Fst1-overexpressing transgenic fish until two weeks post-hatch (Table 2, or via whole mount in situ (data not shown)). However, similar to the results obtained in mice overexpressing $F s t$ in skeletal muscle [10], muscle growth in adult transgenic zebrafish did increase compared with non-transgenic control fish, indicating increased muscle mass. We found no difference in body weight between transgenic and control animals at the hatching stage, and only a moderate increase in body weight in hemizygous Fst1-overexpressing transgenic fish (Table 2). In contrast to the giant zebrafish phenotype obtained by the ubiquitous inhibition of myostatin activity through egg microinjection, muscle specific inhibition of myostatin or other potential regulatory TGF- $\beta$ members using the transgenic method seems to show mild effects on body growth. This might be related to late Fst 1 overexpression from the Mylz2 promoter, whose transcription is initiated only from $20 \mathrm{hpf}$ [13]. It might also suggest that fish myostatin has a substantial body mass regulation function beyond that in muscle tissue. It has been speculated that Fst1 might block other Fst-sensitive ligands expressed in muscle tissue, such as growth and differentiation factor-11 (GDF-11, 11, 7, 8). Previous studies demonstrated that $F s t$ transgenic mice had larger muscle mass increases than transgenic mice overexpressing the dominant negative form the activin receptor $\mathrm{B}$ and myostatin prodomain $[10,17]$. The expression level of $M y o D$ increased in our adult mylz2:Fst1 transgenic fish, which was not observed in myostatin prodomain-overexpressing transgenic zebrafish [2]. Strong muscling in our mylz2:Fst1 transgenic fish was observed in our histological analysis. Phenotypes related to muscle growth seemed more significant in our Fstl overexpressing fish compared to the transgenic zebrafish expressing the myostatin prodomain [2]. This might reflect the stronger effect of Fst1 on muscle growth promotion than the myostatin prodomain protein, because Fst has been suggested to be involved in the inhibition of TGF- $\beta$ members other than myostatin $[7,18]$.

Lack of myostatin could lead to an enhanced level of Pax7 expression or an increased number of Pax7-positive muscle precursor cells. It has been suggested that myostatin signals through Pax7 to regulate the pool of muscle precursors [1]. In the present study, we demonstrated that $\operatorname{Pax} 7$ expression in skeletal muscle was enhanced by Fstl overexpression (Figure 2). Compared with the $10 \%$ increase in myofiber number in myostatin prodomain protein transgenic zebrafish described by $\mathrm{Xu}$ et al. [2], Fstl-overexpressing transgenic fish exhibited a $33 \%$ increase in numbers of fibers. The proportion of small-diameter fibers in our Fst1 transgenic zebrafish is higher than that observed in their control siblings (Figure 3), indicating enhanced post-hatch myogenic proliferation or stratified hyperplasia. However, we observed a $<10 \%$ body weight increase and no obvious fish size difference between Fstl transgenic fish and controls. This might be because of the non-homozygous background of the transgenic fish that we analyzed. Fst overexpressing mice showed reproductive difficulties [19], and we also encountered reproductive difficulties in our Fst 1 transgenic zebrafish. We have not yet been able to obtain homozygous Fst 1 transgenic zebrafish. This might be because of the different mechanisms adopted for modulation of postnatal muscle growth between teleosts and amniotes, in terms of the contributions of hypertrophy or hyperplasia to muscle growth [20]. Muscle mass growth in vertebrates increases by the recruitment of new muscle fibers (hyperplasia), as well as by increasing the size of already existing fibers (hypertrophy) [20,21]. However, small and slowgrowing fish, like zebrafish, largely rely on stratified hyperplasia; this could explain the low capacity of the Fst transgenic fish to further increase their body size, even with significantly increased stratified hyperplasia.

The production of an F0 generation of Fst-overexpressing transgenic trout was recently reported [22]. Fst overexpression in the muscle of the F0 transgenic trout resulted in increased muscling, almost exclusively by muscle fiber hyperplasia. Our observation of the effect of Fst overexpression on muscle mass is similar to previous studies on promyostatin transgenic zebrafish and $F$ st transgenic trout [2,22]. Nevertheless, the present study demonstrated, in vivo, that muscle-specific Fst1 overexpression in zebrafish could enhance muscle growth by upregulating the expression of myogenic factors and enhancing myofiber hyperplasia, probably via negative regulation of the function(s) of myostatin and/or other members of the TGF- $\beta$ super-family.

The authors thank Dr. Vladimir Korzh (Institute of Molecular and Cell Biology, Singapore) and Dr. Gong ZhiYuan (Department of Biological Sciences, Singapore) for the generous gifts of the Tol2-ET trap and Mylz2:EGFP plasmids. This work was supported by the National Natural Science Foundation of China (Grant No. 30671600) and the National Basic Research Program of China (Grant Nos. 2010CB126302, 2009CB118701).

1 McFarlane C, Hennebry A, Thomas M, et al. Myostatin signals through Pax7 to regulate satellite cell self-renewal. Exp Cell Res, 2008, 314: 317-329

$2 \mathrm{Xu} \mathrm{C}, \mathrm{Wu} \mathrm{G}$, Zohar Y, et al. Analysis of myostatin gene structure, expression and function in zebrafish. J Exp Anim, 2003, 206: 4067-4079

3 Amali A A, Lin C J, Chen Y H, et al. Up-regulation of muscle-specific transcription factors during embryonic somitogenesis of zebrafish (Danio rerio) by knock-down of myostatin-1. Dev Dyn, 2004, 229: 847-856

4 Acosta J, Carpio Y, Borroto I, et al. Myostatin gene silenced by RNAi show a zebrafish giant phenotype. J Biotech, 12005, 19: 324-331

5 Kozaki K, Ouchi Y. Activin/follistatin and atherosclerosis-a review. J Atheroscler Thromb, 1998, 5: 36-40

6 Hill J J, Davies M V, Pearson A A, et al. The myostatin propeptide and the follistatin-related gene are inhibitory binding proteins of my- 
ostatin in normal serum. J Biol Chem, 2002, 277: 40735-40741

7 Lee S J. Regulation of muscle mass by myostatin. Annu Rev Cell Dev Biol, 2004, 20: 61-86

8 Biga P R, Roberts S B, Iliev D B, et al. The isolation, characterization, and expression if a novel GDF11 gene and a second myostatin form in zebrafish, Danio rerio. Comp Biochem Physiol B, 2005, 141: 218-230

9 Matzuk M M, Lu N, Vogel H, et al. Multiple defects and perinatal death in mice deficient in follistatin. Nat, 1995, 374: 360-363

10 Lee S J, McPherron A C. Regulation of myostatin activity and muscle growth. Proc Natl Acad Sci USA, 2001, 98: 9306-9311

11 Bellinge R H S, Liberies D A, Iaschi S P A, et al. Myostatin and its implications on animal breeding: a review. Anim Genet, 2004, 36: 1-6

12 Macqueen D J, Johnston I A. Evolution of follistatin in teleosts revealed through phylogenetic, genomic and expression analyses. Dev Genes Evol, 2008, 218: 1-14

$13 \mathrm{Ju} \mathrm{B}$, Chong S W, He J, et al. Recapitulation of fast skeletal muscle development in zebrafish by transgenic expression of GFP under the mylz2 promoter. Dev Dyn, 2003, 227: 14-26

$14 \mathrm{Li} \mathrm{X}, \mathrm{He} \mathrm{J}, \mathrm{Hu} \mathrm{W}$, et al. The essential role of endogenous ghrelin in growth hormone expression during zebrafish adenohypophysis development. Endocrinol, 2009, 150: 2767-2774

15 Parinov S, Kondrichin I, Korzh V, et al. Tol2 transposon-mediated enhancer trap to identify developmentally regulated zebrafish genes in vivo. Dev Dyn, 2004, 231: 449-459

16 Bauer H, Meier A, Hild M, et al. Follistatin and noggin are excluded from zebrafish organizer. Dev Biol, 1998, 204: 488-507

17 Zhu X, Hadhazy M, Wehling M, et al. Dominant negative myostatin produces hypertrophy without hyperplasia in muscle. FEBS Let, 2000, 474: 71-75

18 Helterline D L I, Garikipati D, Stenkamp D L, et al. Embryonic and tissue-specific regulation of myostatin-1 and -2 gene expression in zebrafish. Gen Comp Endocrionl, 2007, 151: 90-97

19 Guo Q, Kumar T R, Woodruff T, et al. Overexpression of mouse follistatin causes reproductive defects in transgenic mice. Mol Endocrinol, 1998, 12: 96-106

20 Biga P R, Goetz F W. Zebrafish and giant danio as models for muscle growth: determinate vs. indeterminate growth as determined by morphometric analysis. Am J Physiol Regul Integr Comp Physiol, 2006, 291: R1327-1337

21 Weatherley A H, Gill H S, Lobo A F. Recruitment and maximal diameter of axial muscle fibres in teleosts and their relationship to somatic growth and ultimate size. J Fish Biol, 1988, 33: 851-859

22 Medeiros E F, Phelps M P, Fuentes F D, et al. Overexpression of follistatin in trout stimulates increased muscling. Am J Physiol Regul Integr Comp Physiol, 2009, 297: R235-242

Open Access This article is distributed under the terms of the Creative Commons Attribution License which permits any use, distribution, and reproduction in any medium, provided the original author(s) and source are credited. 